\title{
Visualization of bone details in a novel photon-counting dual-source CT scanner-comparison with energy-integrating CT
}

\author{
Stefanie J. Bette ${ }^{1} \cdot$ Franziska M. Braun ${ }^{1} \cdot$ Mark Haerting $^{1} \cdot$ Josua A. Decker $^{1}$ - Jan H. Luitjens ${ }^{1}$. \\ Christian Scheurig-Muenkler ${ }^{1} \cdot$ Thomas J. Kroencke $^{1} \cdot$ Florian Schwarz $^{1}$ [D
}

Received: 27 September 2021 / Revised: 25 October 2021 / Accepted: 27 October 2021 / Published online: 22 December 2021

(c) The Author(s) 2021

\begin{abstract}
Objectives Photon-counting detector CT (PCD-CT) promises a leap in spatial resolution due to smaller detector pixel sizes than implemented in energy-integrating detector CTs (EID-CT). Our objective was to compare the visualization of smallest bone details between PCD-CT and EID-CT using a mouse as a specimen.

Materials and methods Two euthanized mice were scanned at a 20-slice EID-CT and a dual-source PCD-CT in single-pixel mode at various $\mathrm{CTDI}_{\mathrm{Vol}}$ values. Image noise and signal-to-noise ratio (SNR) were evaluated using repeated ROI measurements. Edge sharpness of bones was compared by the maximal slope within CT value plots along sampling lines intersecting predefined bones of the spine. Two readers evaluated bone detail visualization at four regions of the spine on a three-point Likert scale at various CTDI $\mathrm{V}_{\mathrm{Vol}}$ 's. Two radiologists selected the series with better detail visualization among each of 20 SNR-matched pairs of EID-CT and PCD-CT series. Results In $\mathrm{CTDI}_{\mathrm{Vol}^{-}}$-matched scans, PCD-CT series showed significantly lower image noise (Noise ${ }_{C T D I=5 m G y}: 16.27 \pm 1.39$ vs. $23.46 \pm 0.96 \mathrm{HU}, p<0.01$ ), higher $\mathrm{SNR}\left(\mathrm{SNR}_{C T D I=5 m G y}: 20.57 \pm 1.89\right.$ vs. $14.00 \pm 0.66, p<0.01$ ), and higher edge sharpness (Edge Slope lumbar spine: $981 \pm 160$ vs. $608 \pm 146 \mathrm{HU} / \mathrm{mm}, p<0.01$ ) than EID-CT series. Two radiologists considered the delineation of bone details as feasible at consistently lower $\mathrm{CTDI}_{\mathrm{Vol}}$ values at PCD-CT than at EID-CT. In comparison of SNR-matched reconstructions, PCD-CT series were still considered superior in almost all cases.

Conclusions In this head-to-head comparison, PCD-CT showed superior objective and subjective image quality characteristics over EID-CT for the delineation of tiniest bone details. Even in SNR-matched pairs (acquired at different CTDI $\mathrm{Vol}_{\mathrm{Vol}}$ 's), PCD-CT was strongly preferred by radiologists.

Key Points

- In dose-matched scans, photon-counting detector CT series showed significantly less image noise, higher signal-to-noise ratio, and higher edge sharpness than energy-integrating detector CT series.

- Human observers considered the delineation of tiny bone details as feasible at much lower dose levels in photon-counting detector CT than in energy-integrating detector CT.

- In direct comparison of series matched for signal-to-noise ratio, photon-counting detector CT series were considered superior in almost all cases.
\end{abstract}

Keywords Multidetector computed tomography $\cdot$ X-ray computed tomography $\cdot$ Bone and bones

\section{Abbreviations}

$\mathrm{CTDI}_{\mathrm{Vol}} \quad$ Volumetric computed tomography dose index

EID-CT Energy-integrating detector CT

FoV Field of view

HU Hounsfield units
PCD-CT Photon-counting detector CT

ROI Region of interest

SNR Signal-to-noise ratio

UHR Ultra-high resolution
Florian Schwarz

florian.schwarz@uk-augsburg.de

1 Clinic for Diagnostic and Interventional Radiology and Neuroradiology, University Hospital Augsburg, Stenglinstr. 2, 86156 Augsburg, Germany

\section{Introduction}

Computed tomography (CT) is the most widely used tomographic imaging modality worldwide due to its short acquisition times, high technical reliability, and ubiquitous 
availability. CT scanners in clinical use today are equipped with energy-integrating detectors (EID) which are based on an indirect detection of X-ray photons after conversion into light in scintillator ceramics.

Very recently, photon-counting detectors (PCDs) have become available for clinical CT. Detecting charge separation in semiconductors induced by X-ray photons, PCDs directly convert X-rays into an electric signal. This confers substantial theoretical benefits such as improved spatial resolution due to smaller detector pixel size, elimination of electronic noise, and intrinsic spectral sensitivity [1,2].

For maximal spatial resolution, a single-pixel acquisition mode is routinely available on the latest PCD-CT models. This mode discerns individual detector pixels and should considerably improve visualization of finest anatomic details such as in bone or in the lungs beyond that reported for earlier PCD-CT prototypes which aggregated two pixels for ultra-high-resolution (UHR) acquisitions [3, 4]. Previous studies on earlier PCD-CT prototypes have compared PCD$\mathrm{CT}$ in UHR mode with EID-CT in phantoms and cadaveric specimen such as wrists and observed higher signal-to-noise ratio (SNR) of PCD-CT in computed tomography dose index $\left(\mathrm{CTDI}_{\mathrm{Vol}}\right)$-equivalent acquisitions hinting at considerable dose-saving potential of the new technology [5, 6]. Uncertainty remains, however, to what extent this SNR advantage translates into improved visualization of anatomic details and whether there is a dose-dependency of this effect.

This study reports our comprehensive analyses of bone detail visualization on a novel dual-source PCD-CT in comparison with a modern EID-CT at various $\mathrm{CTDI}_{\mathrm{Vol}}$ values using a mouse as a specimen. Because of the very small size of its skeleton, highly standardizable skeletal reference regions of different inherent complexity could be defined making the mouse an ideal model for our analysis. We systematically quantified image noise, signal-to-noise ratio, and edge sharpness of bones and compared visualization of bone details at different CTDI $_{\mathrm{Vol}}$ 's between PCD-CT and EID-CT. Finally, observer preference in SNR-matched pairs of PCD$\mathrm{CT}$ and EID-CT series was evaluated.

\section{Materials and methods}

\section{Scanning of specimen}

Two euthanized mice were purchased from a pet food store and positioned inside a sealed plastic box for CT scanning. By changing the position of the box and the table height, the mice were aligned in the isocenter and perpendicularly to the patient table.

The mice were sequentially scanned on two CT scanners (A: a 20-slice MDCT with energy-integrating detector (EID-CT): Somatom AS20, Siemens Healthineers, Erlangen,
Germany; B: a novel photon-counting dual-source CT, NAEOTOM Alpha, Siemens Healthineers (PCD-CT)) using protocols optimized for bone imaging and identical computed tomography dose index settings $\left(\mathrm{CTDI}_{\mathrm{vol}(32 \mathrm{~cm})}\right)$ at $120 \mathrm{kV}$. For EID-CT, collimation was $0.6 \times 20$ with a rotation time of $1.0 \mathrm{~s}$ and a spiral pitch of 0.8 . For PCD-CT, collimation was $0.2 \times 120$ with a rotation time of $0.5 \mathrm{~s}$ and a spiral pitch of 0.2. For PCD-CT acquisitions, the routinely available single-pixel acquisition mode was selected. Scans were performed sequentially at different $\mathrm{CTDI}_{\mathrm{Vol}}$ 's ranging from 2.2 to $20 \mathrm{mGy}$. Tube modulation was disabled for all scans.

\section{Image reconstruction and postprocessing}

For comparison between scanners, kernels with high spatial resolution and closest similarity in modulation transfer function were selected (B75h for EID-CT and Hr68 for PCD-CT, data provided by Siemens Healthineers). For PCDCT, additional reconstructions using a Hr98 Kernel were generated for illustration purposes only. All reconstructions used filtered back projection with a quadratic field of view $(11.4 \mathrm{~cm})$ and a matrix of $512 \times 512$.

For noise measurements, datasets were reconstructed with 1-mm slice thickness and 1-mm increment. For the evaluation of bone detail visualization, the minimal available slice thickness was chosen: $0.6 \mathrm{~mm}$ with an increment of $0.1 \mathrm{~mm}$ for EID-CT and $0.2 \mathrm{~mm}$ with an increment of $0.1 \mathrm{~mm}$ for PCD-CT, respectively. For better comparability, 0.6-mm EID-CT images were reformatted to a slice thickness of $0.2 \mathrm{~mm}$ with $0.1-\mathrm{mm}$ increment using commercially available software (SyngoVia VB60, Siemens Healthineers). Furthermore, identical coronal reformations of the cervical spine were generated with a slice thickness of $0.6 \mathrm{~mm}$ and an increment of $0.1 \mathrm{~mm}$ (Fig. 1, Video 1).

\section{Quantification of image noise, signal-to-noise ratio, and edge sharpness}

Image noise was measured using the open-source software Fiji [7]. Three identical regions of interest (ROIs) were defined in a large volume of air and copied to all 1.0-mm series. Standard deviation of CT values in air was used to approximate image noise and reported as mean of the measurements in the three ROIs.

SNR was quantified using the ratio between the CT values in bone and the standard deviation of CT values in air on $0.2-\mathrm{mm}$ series: four $1.5 \mathrm{~mm}^{2}$ ROIs were defined on parasagittal planes in the lumbar vertebrae L2 and L3 and three large ROIs within air. Mean CT values of bone and mean SD of CT values in air were recorded.

Edge sharpness of bone surfaces was quantified using $\mathrm{CT}$ value plots along two sampling lines manually drawn at defined positions on the $0.2-\mathrm{mm}$ reconstructions: in 


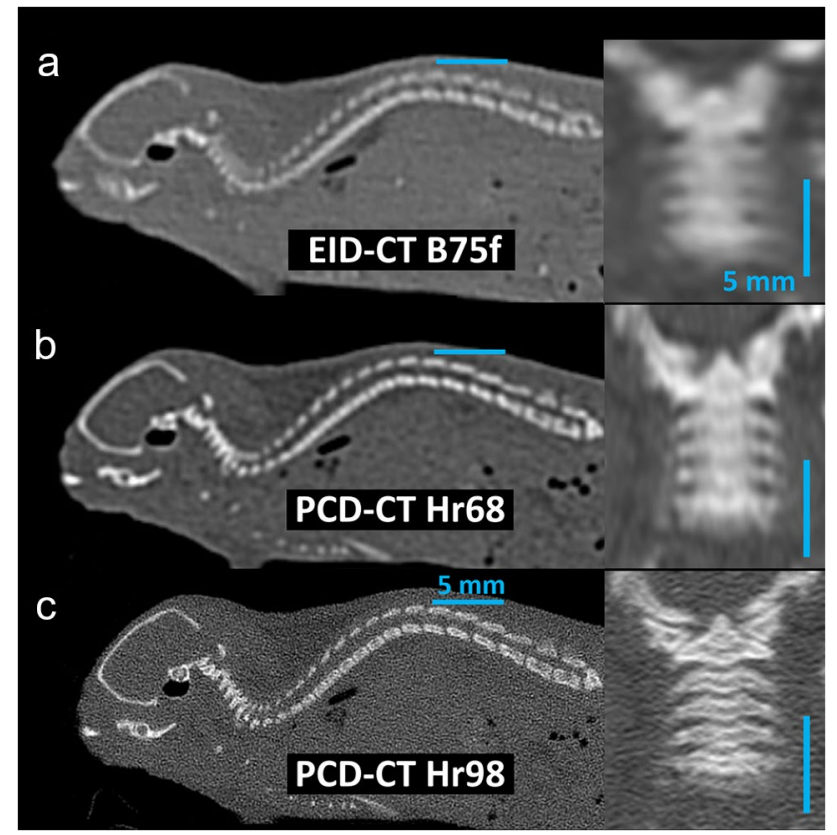

Fig. 1 Reconstructed series used for analysis. Sagittal reconstructions of the whole spine (slice thickness: $0.2 \mathrm{~mm}$ ) and coronal reformations of the cervical spine (slice thickness: $0.6 \mathrm{~mm}$ ) at EID-CT B75f (a), PCD-CT Hr68 (b), and PCD-CT Hr98 (c) at $\mathrm{CTDI}_{\mathrm{Vol}}=15 \mathrm{mGy}$. $\mathrm{CTDI}_{\mathrm{Vol}}$, volumetric CT dose index; EID-CT, energy-integrating detector CT; PCD-CT, photon-counting detector CT; B75h, Hr68, and $\mathrm{Hr} 98$ are abbreviations for specific reconstruction algorithms

parasagittal planes along the cervical spine axis, intersecting the transverse processes; and in the median plane, perpendicularly to the thoracic or lumbar spinal canal intersecting the dorsal lamina. For the cervical spine, three measurements of edge sharpness of the transverse processes of $\mathrm{C} 2$ and $\mathrm{C} 3$ were performed for each $\mathrm{CTDI}_{\mathrm{Vol}}$ value. For the thoracic and lumbar spine, three measurements were performed at each of six vertebrae (Th11-L4) for each $\mathrm{CTDI}_{\mathrm{Vol}}$ value. As a measure for edge sharpness, the slope of the CT value plot at the bone surface was used, defined as the difference of CT values per millimeter:

Edge Slope $=\frac{\mid \Delta \text { CT Values }(\mathrm{HU}) \mid}{\text { Length }(\mathrm{mm})}$

\section{Evaluation of bone detail visualization}

Using the 0.2-mm reconstructions and the 0.6- $\mathrm{mm}$ coronal reformations of the cervical spine, two radiologists semiquantitatively evaluated four defined anatomic regions: (I) lumbar spine: spinal canal; (II) lumbar spine: transverse foramen; (III) cervical spine: transverse processes; and (IV) cervical spine: intervertebral discs. Radiologists assessed the visualization of details in consensus on a 3-point Likert

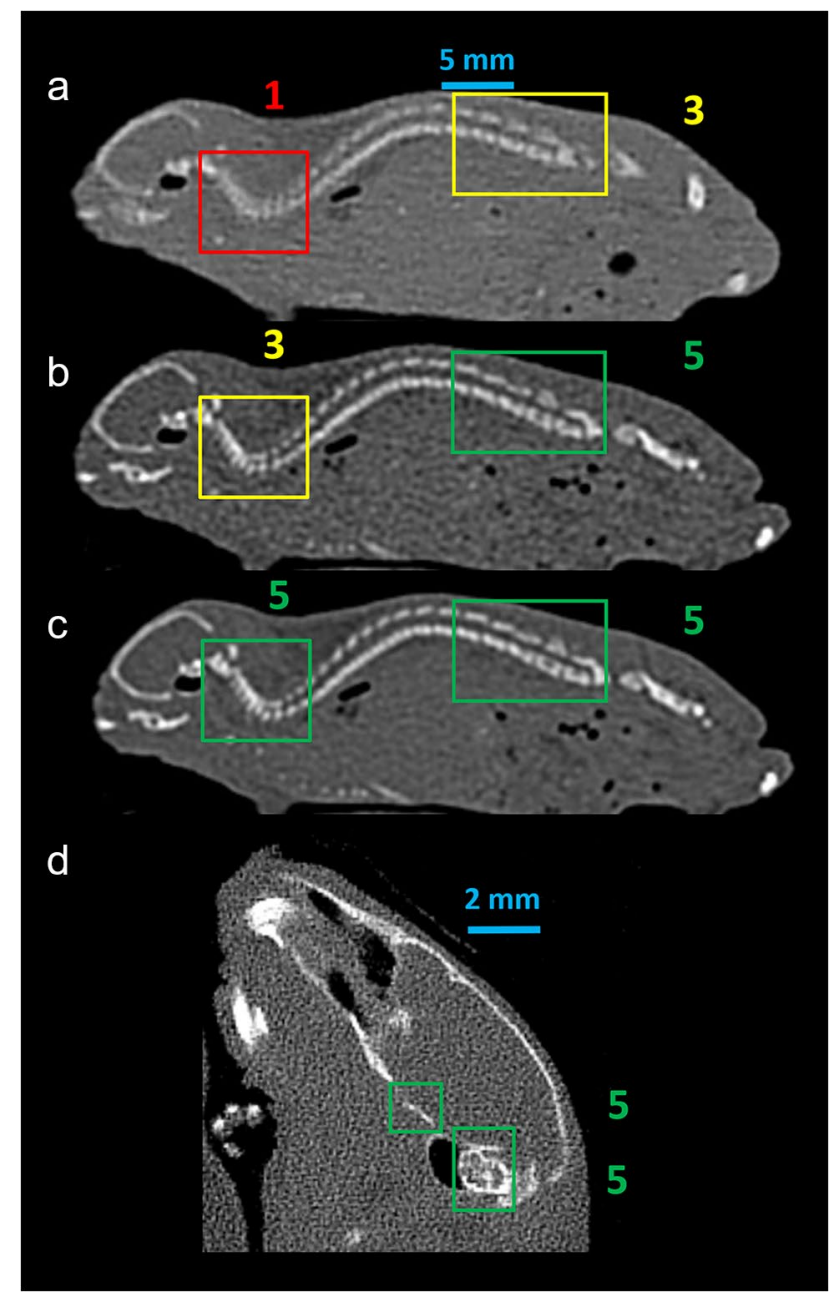

Fig. 2 Semiquantitative analysis of bone detail visualization. Examples for semiquantitative analysis of bone detail visualization. $1=$ no delineation, 2 = unsharp delineation, 3 = sharp delineation. a: EID-CT at $\mathrm{CTDI}_{\mathrm{Vol}}=5 \mathrm{mGy}, \mathbf{b}$ : PCD-CT at $\mathrm{CTDI}_{\mathrm{Vol}}=3 \mathrm{mGy}$, c: PCD-CT at $\mathrm{CTDI}_{\mathrm{Vol}}=10 \mathrm{mGy} . \mathrm{CTDI}_{\mathrm{Vol}}$, volumetric CT dose index; EID-CT, energy-integrating detector $\mathrm{CT}$; PCD-CT, photon-counting detector CT

scale $(1=$ no delineation, $2=$ unsharp delineation, $3=$ sharp delineation, Fig. 2). Readers were blinded towards the scanner and all dose settings and datasets were presented in random order.

\section{Evaluation of observer preference in SNR-matched pairs}

To validate our results from SNR analyses, 20 SNR-matched doubles of EID-CT and PCD-CT series were generated and presented to two readers in random order with the task of selecting the reconstruction with better detail visualization.

Supplemental Fig. 1 shows a flow chart for our study including data acquisition, reconstruction, postprocessing, and data analysis. 


\section{Statistical analysis}

Continuous variables are reported as mean \pm standard deviation and evaluated for normal distribution using Kolmogorov-Smirnov tests. Differences between both groups (EID-CT and PCD-CT) were analyzed by $t$-tests for independent samples if variables were normally distributed. Otherwise, non-parametric tests were used. To test the significance in the difference of proportions, the chi-squared test was applied. A $p$ value $<0.05$ was defined as significant. All analyses were performed on IBM Statistics SPSS 26.0.

\section{Results}

\section{Image noise and signal-to-noise ratio}

Image noise was significantly lower in PCD-CT reconstructions compared to EID-CT reconstructions at all $\mathrm{CTDI}_{\mathrm{Vol}}$ values (all $p$ 's $<0.05$, Fig. 3A, Supplemental Table 1). Outperformance of PCD-CT consistently increased with $\mathrm{CTDI}_{\mathrm{Vol}}$ and ranged from $27.9 \%$ (8.92/31.93; PCD-CT: 23.01 HU vs. EID-CT: $31.93 \mathrm{HU}$, absolute difference $8.92 \mathrm{HU}$ ) at a $\mathrm{CTDI}_{\mathrm{Vol}}$ of $2.2 \mathrm{mGy}$ to $37.3 \%(5.74 / 15.37$; PCD-CT: $9.63 \mathrm{HU}$ vs. EID-CT: $15.37 \mathrm{HU}$, absolute difference $5.74 \mathrm{HU}$ ) at a $\mathrm{CTDI}_{\mathrm{Vol}}$ of $20 \mathrm{mGy}$.

Likewise, SNR was significantly higher in PCD-CT series compared to EID-CT at all $\mathrm{CTDI}_{\mathrm{Vol}}$ values (all $p$ 's $<0.01$, Fig. 3b, Supplemental Table 2). Relative outperformance of PCD-CT series also consistently increased from 18.7\% (2.37/12.7; PCD-CT: 12.7 vs. EID-CT: 10.3, absolute difference 2.37 ) at a $\mathrm{CTDI}_{\mathrm{Vol}}$ of $2.2 \mathrm{mGy}$ to 36.3\% (14.11/38.87; PCD-CT: 38.87 vs. EID-CT: 24.76, absolute difference 14.11) at a $\mathrm{CTDI}_{\mathrm{Vol}}$ of $20 \mathrm{mGy}$.

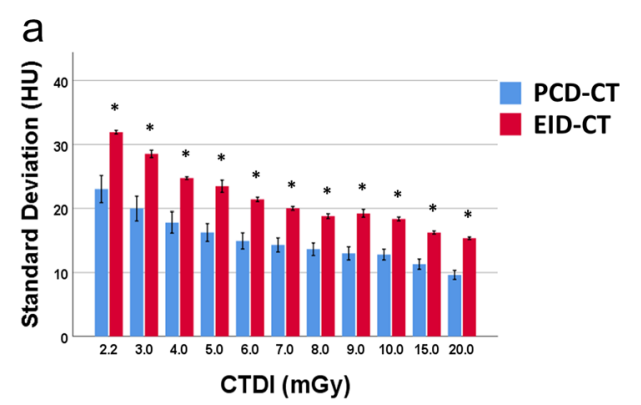

Fig. 3 Comparison of image noise and SNR. (a) Image noise measured as standard deviation of CT values of air at various $\mathrm{CTDI}_{\mathrm{Vol}}$ values. (b) Signal-to-noise ratio for various $\mathrm{CTDI}_{\mathrm{Vol}}$ values.; ${ }^{*} p$

\section{Edge sharpness}

Quantitative analyses of edge sharpness revealed significant differences between EID-CT and PCD-CT with consistently steeper slopes for PCD-CT for all $\mathrm{CTDI}_{\mathrm{Vol}}$ values (all $p$ 's $<0.05$, Fig. 4, Supplemental Table 3). At the lumbar spine, slopes had a range of 543-723 HU/mm for EID-CT and of 940-1042 HU/mm for PCD-CT. At the cervical spine, slopes had a range of 296-1170 HU/mm for EID-CT and 1126-1860 HU/mm for PCD-CT, respectively. As expected, there was no consistent relationship between $\mathrm{CTDI}_{\mathrm{Vol}}$ values and measured slopes so average values across measurements at various $\mathrm{CTDI}_{\mathrm{Vol}}$ 's were used.

\section{Bone detail visualization}

At the cervical spine, the clear visualization of intervertebral discs required a $\mathrm{CTDI}_{\mathrm{Vol}}$ of $20 \mathrm{mGy}$ and $\geq 4 \mathrm{mGy}$ for EIC-CT and PCD-CT, respectively. Likewise, sharp delineation of transverse processes required $\geq 10 \mathrm{mGy}$ and $\geq 4 \mathrm{mGy}$ for EIC-CT and PCD-CT. At the lumbar spine, clear visualization of transverse foramina was observed at $\mathrm{CTDI}_{\mathrm{Vol}}$ values $\geq 6 \mathrm{mGy}$ and $\geq 3 \mathrm{mGy}$ for EID-CT and PCD-CT, whereas sharp delineation of the lumbar spinal canal required $\mathrm{CTDI}_{\mathrm{Vol}}$ values $\geq 4 \mathrm{mGy}$ for EID-CT but was observed at all analyzed $\mathrm{CTDI}_{\mathrm{Vol}}$ values for PCD-CT (cervical spine: Fig. 5; Video 1 lumbar spine: Supplemental Fig. 2).

Additional $\mathrm{Hr} 98$ reconstructions were performed for PCD-CT. These reconstructions are routinely available at PCD-CT single-pixel mode acquisitions and exhibit supreme spatial resolution and sharp delineation of tiny bone details. As these are not available for EID-CT, they were not used for comparison between scanners. Figure 5 and Video 1 demonstrate the cervical spine in coronal 6-mm reformations as examples of bone detail visualization for EID-CT and PCD$\mathrm{CT}$ (Hr68 and Hr98) at different $\mathrm{CTDI}_{\mathrm{Vol}_{\mathrm{l}}}$ values.

b

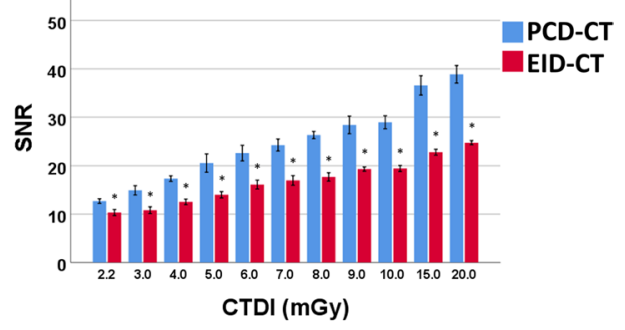

value $<0.05$. $\mathrm{CTDI}_{\mathrm{Vol}}$, volumetric CT dose index; EID-CT, energyintegrating detector CT; HU, Hounsfield units; PCD-CT, photoncounting detector $\mathrm{CT}$; SNR, signal-to-noise ratio 
Fig. 4 Comparison of edge sharpness. Examples of the quantification of edge sharpness. The first row (a) shows cervical spine at PCD-CT $\left(\mathrm{CTDI}_{\mathrm{Vol}}=10 \mathrm{mGy}\right)$ with corresponding plots demonstrating slopes of $\mathrm{CT}$ values. Decrease and increase of CT values is highlighted in red/ green. The second row (b) shows cervical spine at EID-CT $\left(\mathrm{CTDI}_{\mathrm{Vol}}=9 \mathrm{mGy}\right)$ with corresponding plots. The third row (c) shows comparisons of edge sharpness between PCD-CT and EID-CT at three distinct anatomic locations (lumbar spine, cervical spine $\mathrm{C} 2$, cervical spine C3). $\mathrm{CTDI}_{\mathrm{Vol}}$, volumetric CT dose index; EID-CT, energyintegrating detector $\mathrm{CT}$; $\mathrm{HU}$, Hounsfield units; PCD-CT, photon-counting detector $\mathrm{CT}$
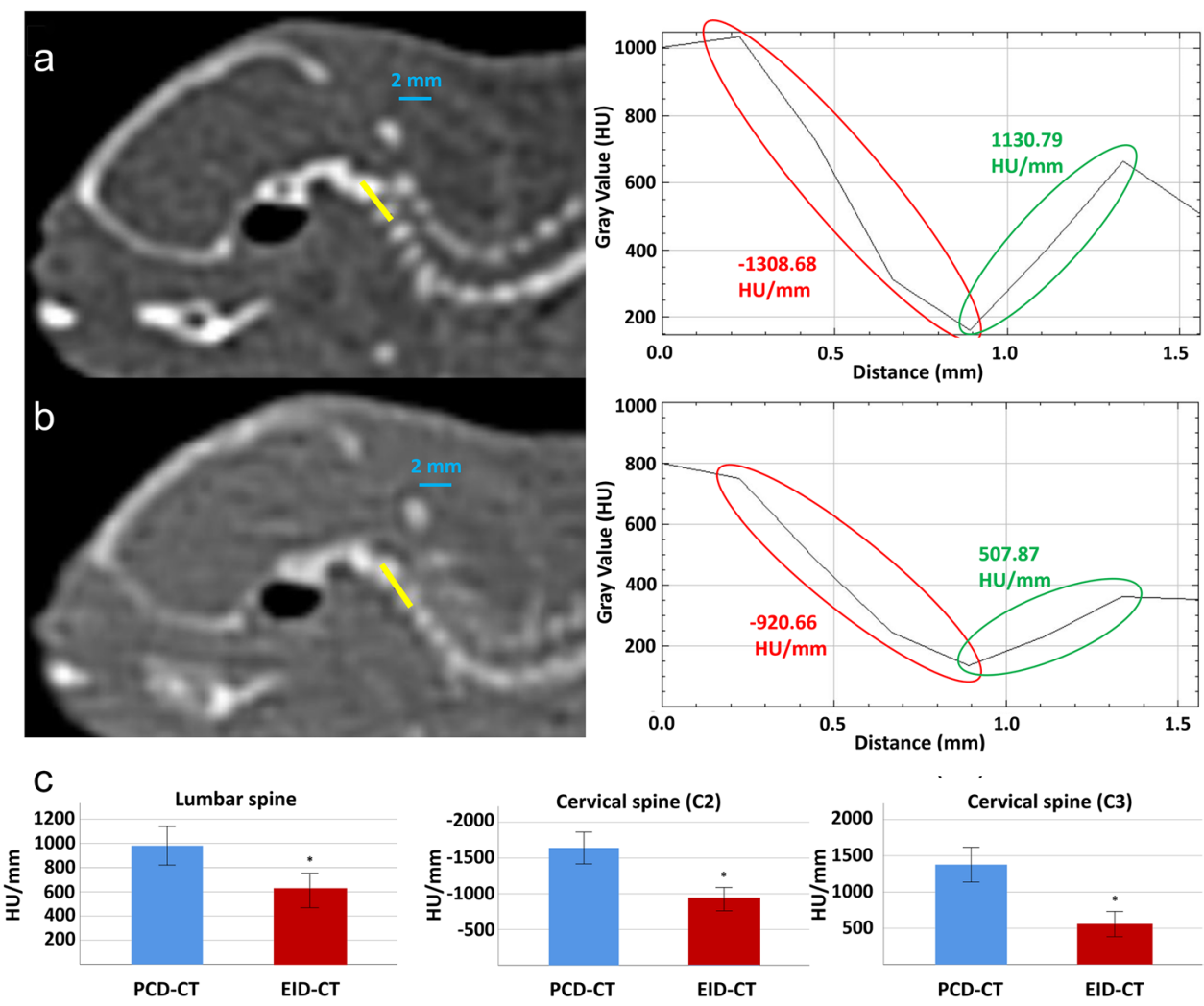

\section{Observer preference in SNR-matched pairs}

SNR-matching produced the following $\mathrm{CTDI}_{\mathrm{Vol}}$-pairs for EID-CT and PCD-CT series, respectively: (20 mGy; $7 \mathrm{mGy}$ ) with SNR of 24.8 and 24.3; (15 mGy; 6 mGy) with SNR of 22.8 vs. 22.6; (10 mGy; 4 mGy) with SNR of 19.4 and 17.4; (6 mGy, $3 \mathrm{mGy}$ ) with SNR of 16.1 and 14.9 and (4 mGy; $2.2 \mathrm{mGy}$ ) with SNR of 12.5 and 12.7, for EID-CT and PCD$\mathrm{CT}$, respectively.

Evaluating SNR-matched pairs for visualization of bone details, reader 1 and reader 2 selected PCD-CT in 19/20 and $18 / 20$ cases, respectively. Both ratios are significantly different from a 50\% ratio (i.e., random choice, both $p$ 's $<0.05$ ).

\section{Discussion}

In this study, we compared the visualization of finest bone details between a novel dual-source PCD-CT in single-pixel mode and EID-CT using a young mouse as a specimen. In dose-matched scans, PCD-CT series showed significantly lower image noise, higher SNR, and higher edge sharpness than EID-CT series. Using comparable linear reconstruction algorithms for both scanners, two radiologists considered the delineation of predefined bone structures (such as transverse foramen) as feasible at consistently lower $\mathrm{CTDI}_{\mathrm{Vol}}$ values at PCD-CT than at EID-CT. In direct comparison of
SNR-matched series, PCD-CT series were still considered superior in almost all cases.

Differences in image noise and SNR were observed across the analyzed $\mathrm{CTDI}_{\mathrm{Vol}}$ spectrum and consistently in favor of PCD-CT and ranged from 27.9 to $37.3 \%$ and from 18.7 to $36.3 \%$ for image noise and SNR, respectively. These observations are consistent with the literature published to date $[5,6,8,9]$. The higher differences in SNR of up to 60\% reported by Grunz et al. [5] might be due to the slightly different definition of image noise in our study. While Grunz et al. used the standard deviation within subcutaneous fat to quantify image noise, we measured standard deviation within a large ROI in adjacent air. Due to the sparsity of subcutaneous fat in young mice, standard deviations within air were much more reproducible. Unlike some of the prior literature, our study solely relied on linear reconstruction algorithms to avoid any confounding by unaddressed differences in iterative algorithms, which might also have contributed to lower differences between PCD-CT and EID-CT in our study.

Our results confirm the dose-saving potential inherent in PCD-CT in single-pixel acquisition mode in comparison with EID-CT. Our results are in line with Grunz et al. [5] in that $\mathrm{CTDI}_{\mathrm{Vol}}$ can be significantly lower in PCD-CT and still yield identical SNRs as EID-CT at higher $\mathrm{CTDI}_{\mathrm{Vol}}$ values, but also extend those of prior studies: PCD-CT datasets with nominally comparable SNR (acquired at much lower 


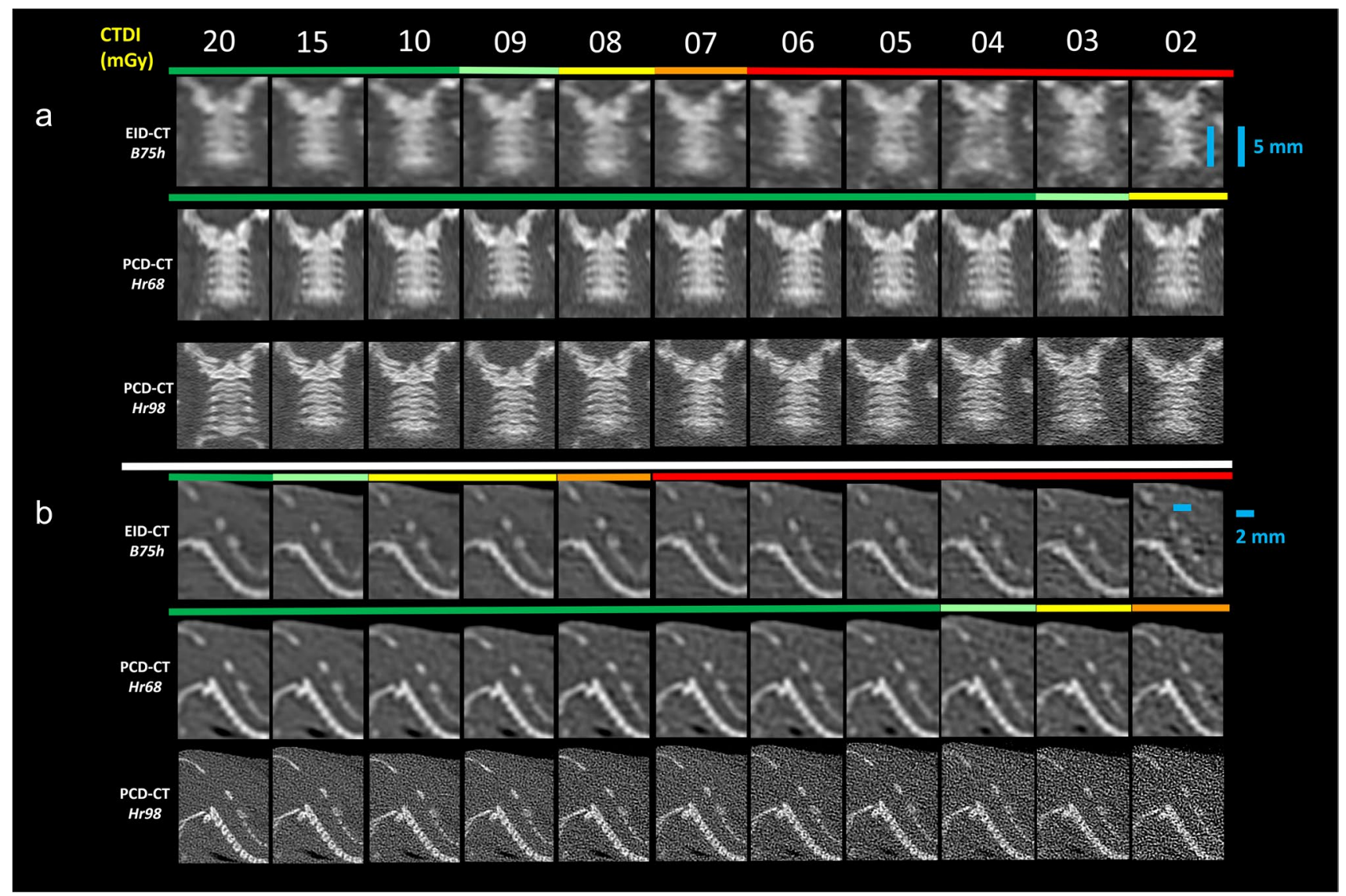

Fig. 5 Semiquantitative evaluation of bone detail visualization on $0.6-\mathrm{mm}$ coronal and $0.2-\mathrm{mm}$ sagittal reformations of the cervical spine. (a) 0.6-mm coronal reformations of the cervical spine for the visualization of the transverse processes. (b) Sagittal 0.2-mm reconstructions of the cervical spine for visualization of the intervertebral discs. Colored lines indicate semiquantitative evaluation of two

$\mathrm{CTDI}_{\mathrm{Vol}}$ values) are still considered superior by observers in the vast majority of cases. This suggests that dose-saving potential should considerably exceed that suggested by SNR difference.

Our study has several limitations. First, it is well known that standard deviations within ROIs incompletely reflect the degree and quality of image noise in a CT dataset and contain no information about its frequency distribution [10]. Nevertheless, this parameter is frequently chosen in image quality studies on clinical CT datasets and some excellent recent work has emphasized the close relationship between this simple parameter and more comprehensive measures of image noise for the types of image noise typically encountered in clinical CT scans $[11,12]$. Second, the mice in our study were scanned without any other attenuating objects in the gantry-except for X-ray transparent casing and the patient table. Therefore, we cannot prove that our results will fully extend to scans of human anatomy with larger diameters or whole patient scans. Yet in those scenarios, scatter readers in consensus for delineation of transverse processes (a) and intervertebral discs (b) at various $\mathrm{CTDI}_{\mathrm{Vol}}$ values; red, no delineation; yellow, unsharp delineation; green, sharp delineation. $\mathrm{CTDI}_{\mathrm{Vol}}$, volumetric CT dose index; EID-CT, energy-integrating detector CT; PCD-CT, photon-counting detector CT; B75h, Hr68, and Hr98 are abbreviations for specific reconstruction algorithms

radiation will be considerably higher; due to the very low sensitivity of PCD-CT for scattered, low-energy photons, difference in image quality in favor of PCD-CT should even be accentuated. Third, we did not employ an UHR comb filter on the EID-CT with which pixel size can be "virtually" reduced thereby increasing spatial resolution. It is conceivable that such an increase in spatial resolution would have increased observer preference for EID-CT images; with the use of an UHR comb, however, dose efficiency of EID-CT would have been considerably worse and in all likelihood much more divergent $\mathrm{CTDI}_{\mathrm{Vol}}$ values would have been compared as SNR equivalent. This would even strengthen our finding of the dose-saving potential of PCD-CT in singlepixel acquisition mode.

In conclusion, our results demonstrate the improved visualization of finest anatomic details using PCD-CT in comparison with EID-CT. This can be attributed to two overlapping effects, namely the better spatial resolution and the reduction of image noise at comparable $\mathrm{CTDI}_{\mathrm{Vol}_{\mathrm{ol}}}$ values. Our 
results are further evidence that this improved visualization does not incur any dose penalty but can be unambiguously measured at comparatively lower radiation doses.

Supplementary Information The online version contains supplementary material available at https://doi.org/10.1007/s00330-021-08441-4.

Funding Open Access funding enabled and organized by Projekt DEAL.

\section{Declarations}

Guarantor The scientific guarantor of this publication is PD Dr. med. Florian Schwarz.

Conflict of interest The authors of this manuscript declare relationships with the following companies:

Thomas Kröncke and Florian Schwarz are (unpaid) members of the "Photon Counting advisory board" of Siemens Healthineers.

The authors of this manuscript declare no relationships with any companies, whose products or services may be related to the subject matter of the article.

Statistics and biometry PD Dr. med. Florian Schwarz, PD Dr. med. Stefanie Bette, and Dr. Josua Decker have significant statistical expertise.

Informed consent Approval from the institutional animal care committee was not required because these were two euthanized mice originally designated to be eaten by snakes. The animals were purchased from a pet food store.

Ethical approval Institutional review board approval was not required because no patients were involved.

\author{
Methodology \\ -prospective. \\ -experimental \\ -performed at one institution.
}

Open Access This article is licensed under a Creative Commons Attribution 4.0 International License, which permits use, sharing, adaptation, distribution and reproduction in any medium or format, as long as you give appropriate credit to the original author(s) and the source, provide a link to the Creative Commons licencwe, and indicate if changes were made. The images or other third party material in this article are included in the article's Creative Commons licence, unless indicated otherwise in a credit line to the material. If material is not included in the article's Creative Commons licence and your intended use is not permitted by statutory regulation or exceeds the permitted use, you will need to obtain permission directly from the copyright holder. To view a copy of this licence, visit http://creativecommons.org/licenses/by/4.0/.

\section{References}

1. Flohr T, Petersilka M, Henning A, Ulzheimer S, Ferda J, Schmidt B (2020) Photon-counting CT review. Phys Medica 79:126-136. https://doi.org/10.1016/j.ejmp.2020.10.030

2. Leng S, Bruesewitz M, Tao S et al (2019) Photon-counting detector CT: system design and clinical applications of an emerging technology. Radiographics 39:729-743. https://doi.org/10.1148/ rg.2019180115

3. Zhou W, Lane JI, Carlson ML et al (2018) Comparison of a photon-counting-detector CT with an energy-integrating-detector CT for temporal bone imaging: a cadaveric study. AJNR Am J Neuroradiol 39:1733-1738. https://doi.org/10.3174/ajnr.A5768

4. Leng S, Rajendran K, Gong $\mathrm{H}$ et al (2018) $150-\mu \mathrm{m}$ spatial resolution using photon-counting detector computed tomography technology: technical performance and first patient images. Invest Radiol 53:655-662. https://doi.org/10.1097/RLI.0000000000 000488

5. Grunz J-P, Huflage H, Heidenreich JF et al (2021) Image quality assessment for clinical cadmium telluride-based photon-counting computed tomography detector in cadaveric wrist imaging. Invest Radiol. https://doi.org/10.1097/RLI.0000000000000789

6. Gutjahr R, Halaweish AF, Yu Z et al (2016) Human imaging with photon counting-based computed tomography at clinical dose levels: contrast-to-noise ratio and cadaver studies. Invest Radiol 51:421-429. https://doi.org/10.1097/RLI.0000000000000251

7. Schindelin J, Arganda-Carreras I, Frise E et al (2012) Fiji: an open-source platform for biological-image analysis. Nat Methods 9:676-682

8. Leng S, Yu Z, Halaweish A et al (2016) Dose-efficient ultrahighresolution scan mode using a photon counting detector computed tomography system. J Med Imaging (Bellingham) 3:043504. https://doi.org/10.1117/1.jmi.3.4.043504

9. Symons R, Reich DS, Bagheri M et al (2018) Photon-counting computed tomography for vascular imaging of the head and neck: first in vivo human results. Invest Radiol 53:135-142. https://doi. org/10.1097/RLI.0000000000000418

10. Boedeker KL, Cooper VN, McNitt-Gray MF (2007) Application of the noise power spectrum in modern diagnostic MDCT: Part I. Measurement of noise power spectra and noise equivalent quanta. Phys Med Biol 52:4027-4046. https://doi.org/10.1088/0031-9155/ $52 / 14 / 002$

11. Christianson O, Winslow J, Frush DP, Samei E (2015) Automated technique to measure noise in clinical CT examinations. AJR Am J Roentgenol 205:W93-W99. https://doi.org/10.2214/AJR.14. 13613

12. Ahmad M, Jacobsen MC, Thomas MA, Chen HS, Layman RR, Jones AK (2020) A benchmark for automatic noise measurement in clinical computed tomography. Med Phys. https://doi.org/10. 1002/mp.14635

Publisher's note Springer Nature remains neutral with regard to jurisdictional claims in published maps and institutional affiliations. 